\title{
Quality control in the diagnosis of Trichuris trichiura and Ascaris lumbricoides using the Kato-Katz technique: experience from three randomised controlled trials
}

Benjamin Speich ${ }^{1,2}$, Said M Ali ${ }^{3}$, Shaali M Ame ${ }^{3}$, Marco Albonico ${ }^{4}$, Jürg Utzinger ${ }^{2,5}$ and Jennifer Keiser ,2* $^{*}$

\begin{abstract}
Background: An accurate diagnosis of soil-transmitted helminthiasis is important for individual patient management, for drug efficacy evaluation and for monitoring control programmes. The Kato-Katz technique is the most widely used method detecting soil-transmitted helminth eggs in faecal samples. However, detailed analyses of quality control, including false-positive and faecal egg count (FEC) estimates, have received little attention.

Methods: Over a 3-year period, within the frame of a series of randomised controlled trials conducted in Pemba, United Republic of Tanzania, 10\% of randomly selected Kato-Katz thick smears were re-read for Trichuris trichiura and Ascaris lumbricoides eggs. In case of discordant result (i.e. positive versus negative) the slides were re-examined a third time. A result was assumed to be false-positive or false-negative if the result from the initial reading did not agree with the quality control as well as the third reading. We also evaluated the general agreement in FECs between the first and second reading, according to internal and World Health Organization (WHO) guidelines.

Results: From the 1,445 Kato-Katz thick smears subjected to quality control, 1,181 (81.7\%) were positive for T. trichiura and 290 (20.1\%) were positive for A. lumbricoides. During quality control, very low rates of false-positive results were observed; $0.35 \%(n=5)$ for T. trichiura and $0.28 \%(n=4)$ for A. lumbricoides. False-negative readings of Kato-Katz thick smears were obtained in $28(1.94 \%)$ and $6(0.42 \%)$ instances for T. trichiura and A. lumbricoides, respectively. A high frequency of discordant results in FECs was observed (i.e. 10.0-23.9\% for T. trichiura, and 9.0-11.4\% for A. lumbricoides).

Conclusions: Our analyses show that the rate of false-positive diagnoses of soil-transmitted helminths is low. As the probability of false-positive results increases after examination of multiple stool samples from a single individual, the potential influence of false-positive results on epidemiological studies and anthelminthic drug efficacy studies should be determined. Existing WHO guidelines for quality control might be overambitious and might have to be revised, specifically with regard to handling disagreements in FECs.
\end{abstract}

Keywords: Soil-transmitted helminths, Diagnosis, Kato-Katz technique, Quality control, False-positive, Faecal egg counts, United Republic of Tanzania

\footnotetext{
* Correspondence: jennifer.keiser@unibas.ch

'Department of Medical Parasitology and Infection Biology, Swiss Tropical and Public Health Institute, Basel, Switzerland

¿University of Basel, Basel, Switzerland

Full list of author information is available at the end of the article
} 


\section{Background}

The common soil-transmitted helminths (i.e. Ascaris lumbricoides, hookworm and Trichuris trichiura) affect approximately 1.5 billion people, cause considerable morbidity and account for an estimated 5.2 million disability adjusted life years (DALYs) [1,2]. An accurate diagnosis is important for the identification of infected individuals, for assessing anthelminthic drug efficacy, and for monitoring the control and elimination of soiltransmitted helminthiasis [3,4]. Currently, the most common diagnostic approach for soil-transmitted helminth infections in epidemiological studies is the copro-microscopic detection of helminth eggs using the Kato-Katz technique $[5,6]$. However, the Kato-Katz technique has limitations in terms of sensitivity, especially in low-intensity settings [3]. The sensitivity of the Kato-Katz technique is increased by analysing multiple thick smears from a single or, ideally, from multiple stool samples [7-15]. The specificity of the Kato-Katz technique is rarely investigated as studies examining the effect of multiple diagnostics usually merge all results to create a composite 'gold' standard, considering all positive results as "true positives" [16]. Henceforth, the possibility of false-positive results has been largely neglected and the frequency of false-positive is unknown [15]. However, assuming 100\% specificity is unrealistic as false-positive results can arise, for example, when debris is confused as helminth eggs or by mistakes recording the data [16]. Tarafder et al. (2010) and Knopp et al. (2014) used Bayesian statistical methods to assess the specificity and sensitivity of the Kato-Katz technique for the diagnosis of soil-transmitted helminth infections [16,17].

In general, when a diagnostic method is used in an epidemiological study, a sub-sample (e.g. 10\%) should be re-examined for quality control to ensure accuracy of the results [18]. However, two recent systematic reviews and meta-analyses revealed that rigorous quality control is the exception rather than the norm in epidemiological studies pertaining to soil-transmitted helminthiases $[19,20]$. A reason for that might be that, until recently, no guidelines were available for judging differences in faecal egg counts (FECs). This gap has been filled; in 2013 the World Health Organization (WHO) released a new guideline that offer some recommendations on how to perform quality control and how to handle differences in FECs [18]. Additionally, at the Swiss Tropical and Public Health Institute (Swiss TPH) an internal guideline has been recently developed. Of note, both of these guidelines distinguish between low and high infection intensities (see Table 1).

In this study, we report new insights from detailed analysis of quality control data obtained over a 3-year period in a series of randomised controlled trials conducted in Pemba, United Republic of Tanzania. We assessed the frequency of false-positive results when using the Kato-Katz technique for the diagnosis of T. trichiura and $A$. lumbricoides. We also analysed the frequency of false-negative results and differences in FECs according to guidelines put forth by the WHO [18] and Swiss TPH.

\section{Methods}

Studies, subjects and quality control samples

In the years 2011, 2012 and 2013, we conducted three randomised controlled trials which evaluated new anthelminthic drugs or drug combinations against soiltransmitted helminths on Pemba Island, United Republic of Tanzania [21-23]. Children attending the schools in Wawi and Al-Sadik (both in 2011), Mchangamdogo and Shungi (both in 2012 and 2013) were invited to participate in the clinical trials. Within the frame of these randomised controlled trials, a total of 14,855 Kato-Katz thick smears were prepared and examined under a microscope at the Public Health Laboratory-Ivo de Carneri (PHL-IdC) by experienced laboratory technicians. Kato-Katz thick smears were prepared according to standard protocols. In brief, we used $41.7 \mathrm{mg}$ templates, and the slides were read within $60 \mathrm{~min}$ to avoid over clearing of hookworm eggs [5,24,25]. Soil-transmitted helminth eggs were counted for each species separately (i.e. A. lumbricoides, hookworm and T. trichiura).

\section{Table 1 Two guidelines how to judge differences in faecal egg counts between the initial reading and the quality control reading of Kato-Katz thick smears}

\begin{tabular}{|c|c|}
\hline Guideline from the World Health Organization (WHO) & $\begin{array}{l}\text { Internal guideline developed at the Swiss Tropical and Public } \\
\text { Health Institute }\end{array}$ \\
\hline $\begin{array}{l}\text { "If the expert identifies a difference in the egg count per gram of stool }{ }^{b} \text { of more } \\
\text { than } 10 \% \text { and more than four eggs, he or she should re-read the slide with } \\
\text { the microscopist and discuss the reasons for the discrepancy". a }[18]\end{array}$ & $\begin{array}{l}\text { Results are considered as inconsistent if there is a difference in } \\
\text { presence/absence of a specific helminth species, or if differences in egg } \\
\text { counts exceed (i) } 10 \text { eggs for Kato-Katz thick smears with } \leq 100 \text { eggs, or } \\
\text { (ii) exceed } 20 \% \text { for Kato-Katz thick smears with more than } 100 \text { eggs. }\end{array}$ \\
\hline
\end{tabular}

${ }^{\mathrm{a}} \mathrm{How}$ to handle differences in presence/absence of helminth eggs is not explicitly stated in the WHO guideline. Therefore we assume that differences in presence/absence of helminth eggs do not require re-reading as long as the difference does not exceed 4 eggs.

${ }^{b}$ To calculate eggs per gram of stool, the egg counts from a single Kato-Katz thick smear are multiplied by a factor of 24 . When multiplying egg counts by a factor of 24 , differences in egg counts of less or equally to four eggs are not possible. Therefore we assume for this current work that the WHO aimed to apply their guideline for egg counts for Kato-Katz thick smears rather than per gram of stool (as it is indicated within a footnote of the WHO document). 
Figure 1 shows the number of Kato-Katz thick smears examined in each of the three trials before and after anthelminthic drug administration. Approximately $10 \%$ of the Kato-Katz thick smears were randomly selected and re-examined for quality control, on a day-to-day basis. As hookworm eggs disintegrate rapidly on Kato-Katz thick smears [24], quality control was restricted to $A$. lumbricoides and $T$. trichiura. Quality control was carried out within 24 hours after preparation of slides.

The results from the initial reading and from the subsequent quality control were compared. In case of discordant results (i.e. positive versus negative for a specific soil-transmitted helminth species and if the investigator judged the difference in FECs subjectively as too large) between the first two readers, a third microscopist was asked to re-examine the respective slide. Quality control (i.e. the second reading) was performed by a senior laboratory technician or by an investigator of the clinical trial. If a third reading was necessary, a third technician who did not previously examine the Kato-Katz thick smear was randomly chosen to re-examine the slide. The only exception was that in cases where a falsepositive result was suspected, the first reader was asked to re-read the slide and show the observed egg to the investigator. All microscopists were blinded to previous results.

\section{Ethical considerations}

For each of the three trials, ethical clearances from the cantonal ethics commission of Basel, Switzerland (EKBB) and from the Ministry of Health and Social Welfare of Zanzibar, United Republic of Tanzania were obtained [21-23]. The trials are registered at Current Controlled Trials (identifiers: ISRCTN08336605, ISRCTN54577342 and ISRCTN80245406). It was emphasised that study participation was voluntary and withdrawal possible at any time without further obligation. At the end of each study, all school-going children were offered albendazole (at a dose of $400 \mathrm{mg}$ ) according to national guidelines $[26,27]$.

\section{Statistical analysis}

Results were classified as false-positive if the original result was positive for a specific soil-transmitted helminth, but the results from the quality control (second reading), as well as from the third reading, were negative. A result was judged as false-negative if the original result was negative, but the quality control as well as the result from the third reading, were positive. We retrospectively calculated (i) the proportion of false-positive results on the overall number of samples; (ii) the proportion of false-positive results among the negative samples; (iii) the proportion of false-negative results among the overall number of samples; and (iv) the proportion of false-

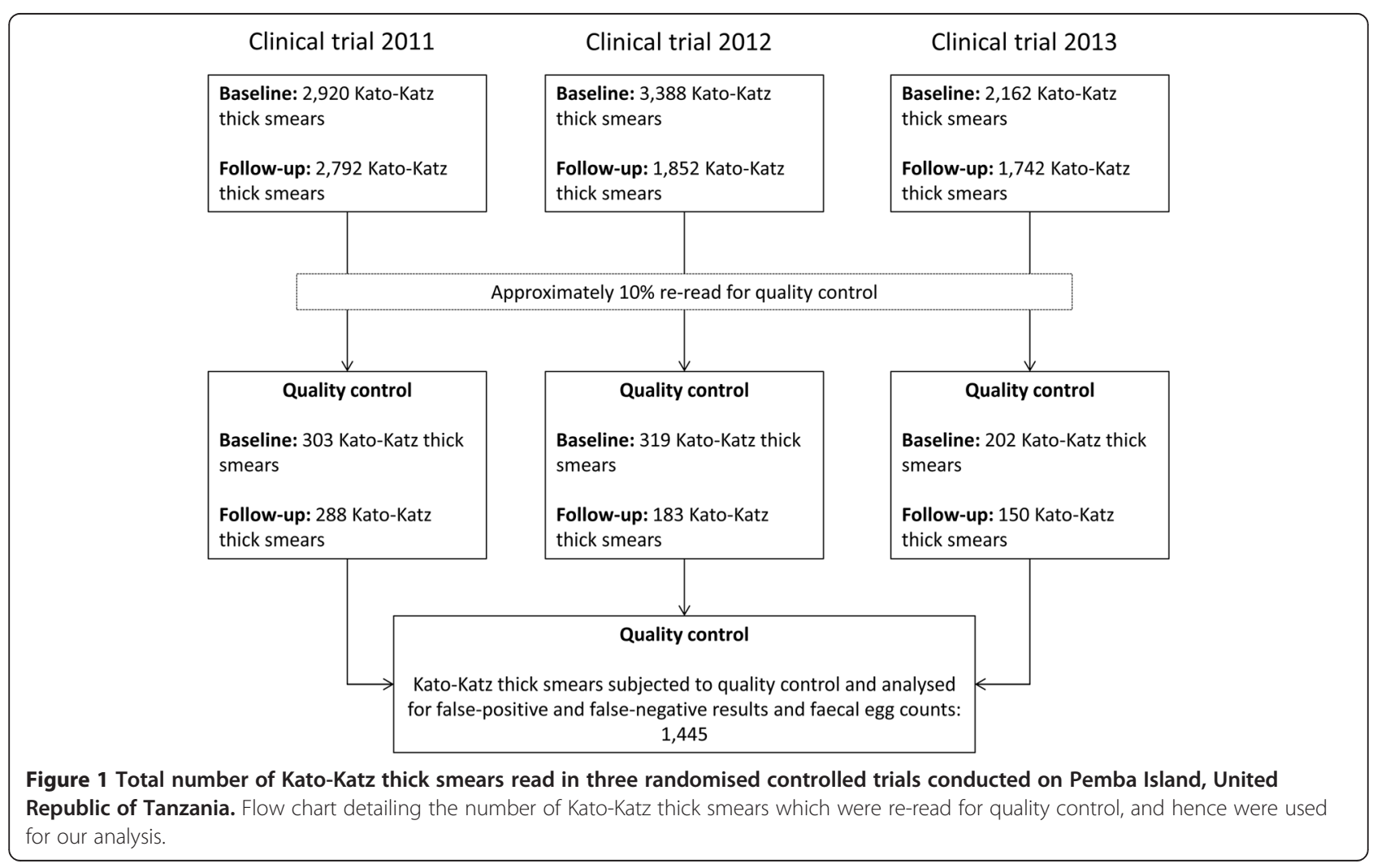


negative results among the positive samples. Speciesspecific differences among (ii) and (iv) were calculated with a two-sample test of proportion. FECs of confirmed false-positive and false-negative readings were descriptively analysed.

FECs from the primary reading were compared to the data from quality control according to two different guidelines put forward by WHO and Swiss TPH. Of note, at the time the clinical trials were implemented, no guidelines on judging FEC differences were available. In contrast to our in-house guideline, it is not explicitly stated in the WHO guideline how to address differences between the observed presence or absence of helminth eggs. Hence, we assumed that, according to WHO guideline, differences in presence/absence of helminth eggs do not require re-reading as long as the difference does not exceed the given range of 4 eggs (see Table 1 ). By comparing the initial readings to the results of the quality control, we retrospectively assessed the proportion of Kato-Katz thick smears which would have required a third re-reading according to the two different guidelines and which were therefore judged as discordant results. In addition, the proportion of agreeing Kato-Katz thick smears among the non-negative slides (i.e. FEC $\geq 1$ either in the initial reading or the quality control) was calculated. These analyses were performed separately for A. lumbricoides and T. trichiura. All data were double entered into an Excel spreadsheet (Microsoft 2010) and cross-checked. Statistical analysis was conducted with Stata version 10.1 software (StataCorp.; College Station, TX, USA).

\section{Results}

A total of 1,445 Kato-Katz thick smears (591 from the first, 502 from the second and 352 from the third trial), all selected at random, were subjected to quality control. The initial reading revealed $1,181(81.7 \%)$ positive slides for T. trichiura and 290 (20.1\%) positive slides for A. lumbricoides. As shown in Table 2, the quality control readings found five false-positive results for $T$. trichiura $(0.35 \%)$ and four false-positive results for A. lumbricoides $(0.28 \%)$. As the prevalence of $T$. trichiura was about four-fold higher than that of A. lumbricoides, there were fewer negative samples, which might have been reported as false-positive. The proportion of false-positive results for $T$. trichiura was higher than for A. lumbricoides; $1.89 \%$ and $0.35 \%$, respectively. Hence, T. trichiura was significantly more often diagnosed as false-positive when analysing only the "true negative" results $(\mathrm{p}<0.01)$.

Among the 1,445 re-examined slides, a total of 28 (1.94\%) and $6(0.42 \%)$ slides were detected as falsenegative for T. trichiura and A. lumbricoides, respectively. The proportion of false-negative results among the positive Kato-Katz thick smears was $2.37 \%$ for T. trichiura and $2.07 \%$ for A. lumbricoides. No significant difference in proportion of false-negative results was detected between T. trichiura and A. lumbricoides $(\mathrm{p}=0.76)$. FECs of the

Table 2 Proportion of false-positive and false-negative results after a second quality control reading of Kato-Katz thick smears (in case of discordant results, slides were read a third time)

\begin{tabular}{|c|c|c|}
\hline & Trichuris trichiura & $\begin{array}{l}\text { Ascaris } \\
\text { lumbricoides }\end{array}$ \\
\hline Total Kato-Katz thick smears read for quality control & 1,445 & 1,445 \\
\hline Positive Kato-Katz thick smears & 1,181 & 290 \\
\hline Negative Kato-Katz thick smears & 264 & 1,155 \\
\hline Percentage of positive Kato-Katz thick smears & $81.7 \%$ & $20.1 \%$ \\
\hline False-positive results ${ }^{\mathrm{a}}$ & 5 & 4 \\
\hline Percentage of false-positive results & $0.35 \%$ & $0.28 \%$ \\
\hline Percentage of false-positive results among negative Kato-Katz thick smears & $1.89 \%^{\mathrm{d}}$ & $0.35 \%^{\mathrm{d}}$ \\
\hline Faecal egg counts of the false-positive Kato-Katz thick smears ${ }^{b}$ & $1,3,4,7,10$ & $2,2,2,12$ \\
\hline False-negative results ${ }^{c}$ & 28 & 6 \\
\hline Percentage of false-negative results & $1.94 \%$ & $0.42 \%$ \\
\hline Percentage of false-negative results among positive Kato-Katz thick smears & $2.37 \%{ }^{\mathrm{e}}$ & $2.07 \%{ }^{\mathrm{e}}$ \\
\hline Faecal egg counts from the quality control of the false-negative Kato-Katz thick smears ${ }^{b}$ & $\begin{array}{l}1,1,1,1,1,1,1,1,1,2,2,2,2,3,3 \\
3,3,3,4,4,5,6,9,10,11,12,15,16\end{array}$ & $1,1,1,1,6,8$ \\
\hline
\end{tabular}

${ }^{a}$ Results were classified as false-positive if the original result was positive for a specific soil-transmitted helminth, but the results from the quality control (second reading), as well as from the third reading, were negative.

${ }^{\mathrm{b}}$ Faecal egg counts per Kato-Katz thick smear.

${ }^{\mathrm{c}}$ Results were classified as false-negative if the original result was negative, but the quality control as well as the result from the third reading, were positive.

${ }^{d}$ Significant difference $(p<0.01)$.

${ }^{\mathrm{e}}$ No significant difference $(p=0.76)$. 
false-positive and false-negative results are listed in Table 2.

Differences in FECs between the initial reading and the quality control are presented in Table 3. From the 1,445 Kato-Katz thick smears read, discrepancies in FECs according to WHO guidelines [18] were detected in $345(23.9 \%)$ and $148(10.2 \%)$ of the Kato-Katz thick smears for T. trichiura and A. lumbricoides, respectively. According to the in-house guideline, differences in FECs were observed on 97 (6.7\%) slides for T. trichiura and on 108 (7.5\%) slides for A. lumbricoides. Additionally, differences in presence versus absence of FECs were observed in $51(3.5 \%)$ and $32(2.2 \%)$ slides for T. trichiura and A. lumbricoides, respectively. According to WHO guidelines, differences in presence versus absence were judged as acceptable as long as they did not exceed 4 eggs per slide, while our in-house guideline would recommend re-reading of the specific Kato-Katz thick smears (see Table 1). Hence, according to our internal guideline, a total of 145 (10.0\%) T. trichiura-positive and 130 (9.0\%) A. lumbricoides-positive slides would have been required to be re-read a third time (Table 3). When excluding all Kato-Katz thick smears which were negative in the initial as well as in the quality control reading, the percentage of disagreeing results increased considerably for both $A$. lumbricoides (i.e. $49.8 \%$ according to WHO guideline and $43.8 \%$ according to Swiss TPH guideline, Table 3) and for T. trichiura (i.e. $29.0 \%$ according to $\mathrm{WHO}$ guideline and $12.2 \%$ according to Swiss
TPH guideline, Table 3). For both soil-transmitted helminth species, the WHO guideline would classify significantly more readings as discordant compared with our in-house guideline $(\mathrm{p}<0.05)$.

\section{Discussion}

The Kato-Katz technique is a widely used method for diagnosing soil-transmitted helminth infections in epidemiological surveys [14]. Usually, multiple Kato-Katz thick smears are examined per study participant to increase diagnostic sensitivity [7-15]. In general, it is assumed that an individual is positive if at least one out of several diagnostic tests revealed a positive result [16]. Thus far, the possibility of false-positive results in the diagnosis of soil-transmitted helminths was largely neglected. In this study we retrospectively calculated the frequency of false-positive diagnoses, facilitated by a detailed analysis of quality control results. We detected only low numbers of false-positive readings ( $T$. trichiura, $\mathrm{n}=5$; A. lumbricoides, $\mathrm{n}=4$ ). When analysing only negative Kato-Katz thick smears, the proportion of falsepositive results was significantly higher for $T$. trichiura (1.89\%) than for A. lumbricoides $(0.35 \%)$. The fact that T. trichiura was more often over-diagnosed than A. lumbricoides might be explained by the smaller shape of its eggs, which might resemble debris in stool more closely. However, we hypothesise that within a single Kato-Katz thick smear, only a low number of debris particles can be confused with helminth eggs. Therefore, the false-

Table 3 Agreement in faecal egg counts (FECs) between initial reading and second quality control reading of Kato-Katz thick smears according to two different guidelines

\begin{tabular}{|c|c|c|c|c|}
\hline & WHO guideline ${ }^{a}$ & & Swiss TPH guidel & $\operatorname{line}^{a}$ \\
\hline & $\begin{array}{l}\text { Trichuris } \\
\text { trichiura }\end{array}$ & $\begin{array}{l}\text { Ascaris } \\
\text { lumbricoides }\end{array}$ & $\begin{array}{l}\text { Trichuris } \\
\text { trichiura }\end{array}$ & $\begin{array}{l}\text { Ascaris } \\
\text { lumbricoides }\end{array}$ \\
\hline Total number of Kato-Katz thick smears (positive/negative) & $1,445(1,189 / 256)$ & $1,445(297 / 1,148)$ & $1,445(1,189 / 256)$ & $1,445(297 / 1,148)$ \\
\hline 1. No. of Kato-Katz thick smears with difference in FECs (\%) & $345(23.9 \%)$ & $148(10.2 \%)$ & $97(6.7 \%)$ & $108(7.5 \%)$ \\
\hline $\begin{array}{l}\text { 2. No. of Kato-Katz thick smears with differences in presence/absence } \\
\text { of helminth eggs (\%) }\end{array}$ & $51(3.5 \%)$ & $32(2.2 \%)$ & $51(3.5 \%)$ & $32(2.2 \%)$ \\
\hline $\begin{array}{l}\text { 3. No. of Kato-Katz thick smears with difference in FECs (\%) among } \\
\text { samples with egg counts }\end{array}$ & $345(29.0 \%)$ & $148(49.8 \%)$ & $97(8.2 \%)$ & $108(36.4 \%)$ \\
\hline $\begin{array}{l}\text { 4. No. of Kato-Katz thick smears with differences in presence/absence } \\
\text { of helminth eggs (\%) among samples with egg counts }\end{array}$ & $51(4.3 \%)$ & $32(10.8 \%)$ & $51(4.3 \%)$ & $32(10.8 \%)$ \\
\hline 1. and/or 2. (\%) & $381(26.4 \%)$ & $164(11.4 \%)$ & $145(10.0 \%)$ & $130(9.0 \%)$ \\
\hline $\begin{array}{l}\text { No. of Kato-Katz thick smears with discrepancies according to respective } \\
\text { guideline }{ }^{b}(\%)\end{array}$ & $345(23.9 \%)$ & $164(11.4 \%)$ & $145(10.0 \%)$ & $130(9.0 \%)$ \\
\hline $\begin{array}{l}\text { 3. and/or } 4 \text {. and proportion as percentage (\%) among Kato-Katz thick } \\
\text { smears with positive egg counts }\end{array}$ & $381(32.0 \%)$ & $164(55.2 \%)$ & $145(12.2 \%)$ & 130 (43.8\%) \\
\hline $\begin{array}{l}\text { No. of Kato-Katz thick smears with discrepancies according to respective } \\
\text { guideline }{ }^{b}(\%) \text { among Kato-Katz thick smears with positive egg counts }\end{array}$ & 345 (29.0\%) & 148 (49.8\%) & 145 (12.2\%) & 130 (43.8\%) \\
\hline
\end{tabular}

${ }^{\mathrm{a}}$ See Table 1 for definition of the WHO and Swiss TPH guidelines.

${ }^{\mathrm{b}}$ The WHO guideline state that differences which exceed $10 \%$ and more than four eggs require re-reading (see Table 1). However, in contrast to the Swiss TPH guideline, differences in presence/absence of helminth eggs are not explicitly stated. Therefore we assumed that differences in presence/absence of helminth eggs do not require re-reading as long as the difference does not exceed four eggs. 
positive Kato-Katz thick smears where several eggs were counted mistakenly (e.g. 10 or 12 eggs; Table 2), might point to another source of error (e.g. writing errors on the entry forms or eggs confused with eggs from different species [16,28]). Figure 2 shows some debris which might resembles an A. lumbricoides egg. Similar as for false-positive results, we suspect that the false-negative Kato-Katz thick smears which contained a larger number of eggs (Table 2) were probably due to writing errors. Another reason might be the tiredness of the readers due to a high number of Kato-Katz thick smears read per day. As a single Kato-Katz thick smear has low sensitivity, the proportion of false-negative diagnostics is usually assessed by examining stool samples with multiple diagnostic techniques, and by analysing multiple stool samples. As this was obviously not done within the current quality control investigation, the true number of false-negative diagnosed individuals is most likely underestimated [10,29].

Even though the proportion of false-positive was relatively low (i.e. $1.89 \%$ for T. trichiura and $0.35 \%$ for $A$. lumbricoides) its impact should not be neglected. While false-negative results can be corrected to some extent by examining multiple KatoKatz thick smears, the probability of false-positive results increases as a function of examining multiple Kato-Katz thick smears [8]. Hence, in studies where multiple Kato-Katz thick smears are examined from each participant, the false-positive rate per individual might not be negligible. This hypothesis is confirmed by a recent study by Tarafder and colleagues (2010) who used Bayesian statistical methods to calculate the specificity of a single and multiple Kato-Katz thick smears. While they reported high specificity for a single Kato-Katz thick smear, specificity decreased by examining multiple Kato-Katz slides [16].

Differences in FECs between the initial and the quality control readings were assessed according to two different guidelines (Table 1). To our knowledge, we evaluated for the first time these guidelines with a large dataset from three randomised controlled trials. A relatively high frequency of discordant results was observed according to both guidelines. This observation indicates that an accurate counting of eggs in studies where high

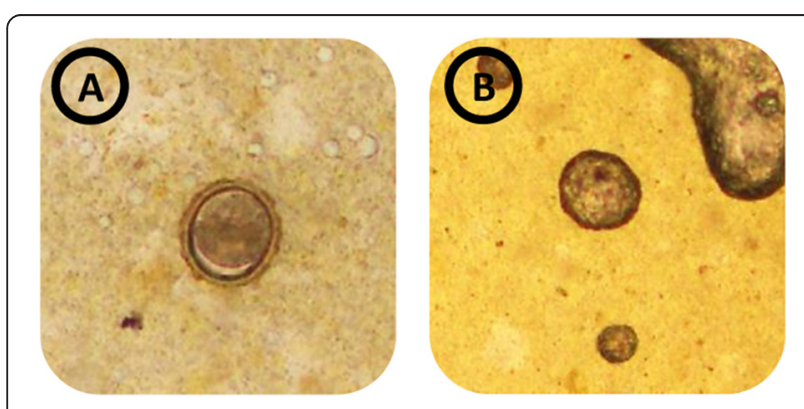

Figure 2 Kato-Katz thick smears with an Ascaris lumbricoides egg (A), as well as debris that resembles an A. lumbricoides egg (B). numbers of Kato-Katz thick smears are read in relatively short time frames is challenging. A particularly large number of disagreeing results was observed for the A. lumbricoides-positive slides. It is not entirely clear, whether A. lumbricoides eggs are generally more difficult to detect or if the high egg counts, which are more common for A. lumbricoides, were responsible for this result $[30,31]$. Given the fact that enumerating soil-transmitted helminth eggs is challenging, one might consider revising the current $\mathrm{WHO}$ guideline, which currently seem to be too firm and therefore might be overambitious. In our opinion another limitation of the WHO guideline is that a difference in presence versus absence of eggs is not considered a discordant result, as long as the FEC differed by not more than 4 eggs (see Table 1 ). However, verification of presence or absence of a helminth infection is crucial, as false-negative or false-positive diagnoses have important ramifications on patient management.

A limitation of our study is that we cannot evaluate the impact of further error sources of false-positive results, as for example sampling errors which can occur when children mix up or even share stool samples. Additionally, the aforementioned guidelines on how to judge and handle FEC differences in quality control had not yet been available at the time the clinical trials were conducted. Therefore, Kato-Katz thick smears with differing FECs were only re-read a third time if the investigator judged the difference in FECs as too large. This was done in a subjective way and not as strictly as might be suggested by the guidelines. Due to this reason, we did not have many third reading results for discordant FECs and could thus only compare the first and the quality control reading. It is important to note that discrepancies in those two readings could have equally arose due to reading errors by the initial microscopist as well as reading errors from the microscopist who conducted the quality control.

It should also be highlighted that our results are not directly transferable to other epidemiological settings. First of all, the technicians from the WHO Collaborating Centre PHL-IdC are highly skilled and they have examined tens of thousands of Kato-Katz thick smears over the past several years. Furthermore, the rigorous implementation of a quality control scheme might have increased the overall quality of the Kato-Katz thick smear readings leading to a low number of false-positive and false-negative readings. However, a high frequency of discordant FECs was observed, even though rigorous quality control was in place and technicians were highly experienced.

\section{Conclusion}

We observed low rates of false-positive results of A. lumbricoides and T. trichiura when using the KatoKatz technique. Our results indicate that especially in a 
setting where soil-transmitted helminth infections are highly prevalent, false-positive results will only have a small effect in overall prevalence estimates. However, in settings with low prevalence where it is challenging to identify infected individuals, false-positive results might influence treatment allocation, results of epidemiological studies and monitoring of control programmes. Examining multiple stool samples from participating individuals will further increase the frequency of false-positive results. Additionally, we have shown that an accurate counting of helminth eggs is a formidable challenge, even for highly skilled technicians. Therefore, the WHO guideline might be too strict and one might additionally cast doubt on the reliability of FECs as well as egg reduction rate, which has recently been proposed as the single most important metrics for assessing anthelminthic drug efficacy. We recommend to validate the current WHO guideline within different settings and to adapt it if the frequency of discordant results remains equally high.

\section{Competing interests}

The authors declare that they have no competing interests.

\section{Authors' contributions}

BS, MA, JU and JK designed the study. BS, SaMA and ShMA generated the data. BS wrote the first draft of the manuscript. MA, JU and JK revised the manuscript. All authors read and approved the final version of the manuscript.

\section{Acknowledgements}

We thank Dr. Peter Steinmann from the Swiss Tropical and Public Health Institute for developing the in-house guideline for handling discrepancies among faecal egg count readings. This work was financially supported by the Medicor Foundation and the Swiss National Science Foundation (Grant no. 320030_149310/1). The funders had no role in study design, data collection and analysis, decision to publish, or preparation of the manuscript.

\section{Author details}

${ }^{1}$ Department of Medical Parasitology and Infection Biology, Swiss Tropical and Public Health Institute, Basel, Switzerland. 'University of Basel, Basel, Switzerland. ${ }^{3}$ Laboratory Division, Public Health Laboratory-Ivo de Carneri, Chake Chake, Tanzania. ${ }^{4}$ Ivo de Carneri Foundation, Milan, Italy. ${ }^{5}$ Department of Epidemiology and Public Health, Swiss Tropical and Public Health Institute, Basel, Switzerland.

Received: 17 December 2014 Accepted: 27 January 2015

Published online: 05 February 2015

\section{References}

1. Murray CJL, Vos T, Lozano R, Naghavi M, Flaxman AD, Michaud C, et al. Disability-adjusted life years (DALYs) for 291 diseases and injuries in 21 regions, 1990-2010: a systematic analysis for the Global Burden of Disease Study 2010. Lancet. 2012;380:2197-223.

2. Pullan RL, Smith JL, Jasrasaria R, Brooker SJ. Global numbers of infection and disease burden of soil transmitted helminth infections in 2010. Parasit Vectors. 2014;7:37.

3. Bergquist R, Johansen MV, Utzinger J. Diagnostic dilemmas in helminthology: what tools to use and when? Trends Parasitol. 2009;25:151-6.

4. McCarthy JS, Lustigman S, Yang GJ, Barakat RM, García HH, Sripa B, et al. A research agenda for helminth diseases of humans: diagnostics for control and elimination programmes. PLoS Negl Trop Dis. 2012;6:e1601.

5. Katz N, Chaves A, Pellegrino J. A simple device for quantitative stool thicksmear technique in schistosomiasis mansoni. Rev Inst Med Trop São Paulo. 1972;14:397-400

6. Speich B, Knopp S, Mohammed KA, Khamis IS, Rinaldi L, Cringoli G, et al. Comparative cost assessment of the Kato-Katz and FLOTAC techniques for soil-transmitted helminth diagnosis in epidemiological surveys. Parasit Vectors. 2010;3:71.

7. Ebrahim A, El-Morshedy H, Omer E, El-Daly S, Barakat R. Evaluation of the Kato-Katz thick smear and formol ether sedimentation techniques for quantitative diagnosis of Schistosoma mansoni infection. Am J Trop Med Hyg. 1997;57:706-8.

8. Utzinger J, Booth M, N'Goran EK, Müller I, Tanner M, Lengeler C. Relative contribution of day-to-day and intra-specimen variation in faecal egg counts of Schistosoma mansoni before and after treatment with praziquantel. Parasitology. 2001;122:537-44.

9. Booth M, Vounatsou P, N'Goran EK, Tanner M, Utzinger J. The influence of sampling effort on the performance of the Kato-Katz technique in diagnosing Schistosoma mansoni and hookworm co-infections in rural Côte d'Ivoire. Parasitology. 2003;127:525-31.

10. Steinmann $P$, Du ZW, Wang $L B$, Wang $X Z$, Jiang JY, Li LH, et al. Extensive multiparasitism in a village of Yunnan province, People's Republic of China, revealed by a suite of diagnostic methods. Am J Trop Med Hyg. 2008;78:760-9.

11. Knopp S, Rinaldi L, Khamis IS, Stothard JR, Rollinson D, Maurelli MP, et al. A single FLOTAC is more sensitive than triplicate Kato-Katz for the diagnosis of low-intensity soil-transmitted helminth infections. Trans R Soc Trop Med Hyg. 2009;103:347-54.

12. Glinz D, Silué KD, Knopp S, Lohourignon LK, Yao KP, Steinmann P, et al. Comparing diagnostic accuracy of Kato-Katz, Koga agar plate, etherconcentration, and FLOTAC for Schistosoma mansoni and soil-transmitted helminths. PLoS Negl Trop Dis. 2010;4:e754.

13. Levallois $P$, Chevalier $P$, Gingras $S$, Déry $P$, Payment $P$, Michel $P$, et al. Risk of infectious gastroenteritis in young children living in Québec rural areas with intensive animal farming: results of a case-control study (2004-2007). Zoonoses Public Health. 2014;61:28-38.

14. Nikolay B, Brooker SJ, Pullan RL. Sensitivity of diagnostic tests for human soil-transmitted helminth infections: a meta-analysis in the absence of a true gold standard. Int J Parasitol. 2014;44:765-74.

15. Speich B, Utzinger J, Marti H, Ame SM, Ali SM, Albonico M, et al. Comparison of the Kato-Katz method and ether-concentration technique for the diagnosis of soil-transmitted helminth infections in the framework of a randomised controlled trial. Eur J Clin Microbiol Infect Dis. 2014;33:815-22.

16. Tarafder MR, Carabin H, Joseph L, Balolong E, Olveda R, McGarvey ST. Estimating the sensitivity and specificity of Kato-Katz stool examination technique for detection of hookworms, Ascaris lumbricoides and Trichuris trichiura infections in humans in the absence of a "gold standard". Int J Parasitol. 2010;40:399-404.

17. Knopp S, Salim N, Schindler T, Karagiannis-Voules DA, Rothen J, Lweno O, et al. Diagnostic accuracy of Kato-Katz, FLOTAC, Baermann, and PCR methods for the detection of light-intensity hookworm and Strongyloides stercoralis infections in Tanzania. Am J Trop Med Hyg. 2014;90:535-45.

18. WHO. Assessing the efficacy of anthelminthic drugs against schistosomiasis and soil-transmitted helminthiases. Geneva: World Health Organization; 2013.

19. Ziegelbauer K, Speich B, Mäusezahl D, Bos R, Keiser J, Utzinger J. Effect of sanitation on soil-transmitted helminth infection: systematic review and meta-analysis. PLoS Med. 2012;9:e1001162.

20. Strunz EC, Addiss DG, Stocks ME, Ogden S, Utzinger J, Freeman MC. Water, sanitation, hygiene, and soil-transmitted helminth infection: a systematic review and meta-analysis. PLoS Med. 2014;11:e1001620.

21. Speich B, Ame SM, Ali SM, Alles R, Hattendorf J, Utzinger J, et al. Efficacy and safety of nitazoxanide, albendazole, and nitazoxanide-albendazole against Trichuris trichiura infection: a randomized controlled trial. PLoS Negl Trop Dis. 2012;6:e1685.

22. Speich B, Ame SM, Ali SM, Alles R, Huwyler J, Hattendorf J, et al. Oxantel pamoate-albendazole for Trichuris trichiura infection. N Engl J Med. 2014;370:610-20

23. Speich B, Ali SM, Ame SM, Bogoch II, Alles R, Huwyler J, et al. Efficacy and safety of albendazole-ivermectin, albendazole-mebendazole, albendazoleoxantel pamoate, and mebendazole against Trichuris trichiura and concomitant soil-transmitted helminth infections: a randomised controlled trial. Lancet Infect Dis. (in press); doi:10.1016/S1473-3099(14)71050-3.

24. Martin LK, Beaver PC. Evaluation of Kato thick-smear technique for quantitative diagnosis of helminth infections. Am J Trop Med Hyg. 1968;17:382-91.

25. Yap P, Fürst T, Müller I, Kriemler S, Utzinger J, Steinmann P. Determining soil-transmitted helminth infection status and physical fitness of schoolaged children. J Vis Exp. 2012;66:e3966.

26. Albonico M, Crompton DWT, Savioli L. Control strategies for human intestinal nematode infections. Adv Parasitol. 1999;42:277-341. 
27. WHO. Model list of essential medicines for children (2nd list, March 2010 update). Geneva: World Health Organization; 2010

28. Steinmann P, Rinaldi L, Cringoli G, Du ZW, Marti H, Jiang JY, et al. Morphological diversity of Trichuris spp. eggs observed during an anthelminthic drug trial in Yunnan, China, and relative performance of parasitologic diagnostic tools. Acta Trop. 2015:141:184-8.

29. Knopp S, Mgeni AF, Khamis IS, Steinmann P, Stothard JR, Rollinson D, et al. Diagnosis of soil-transmitted helminths in the era of preventive chemotherapy: effect of multiple stool sampling and use of different diagnostic techniques. PLoS Negl Trop Dis. 2008;2:e331.

30. Bethony J, Brooker S, Albonico M, Geiger SM, Loukas A, Diemert D, et al. Soil-transmitted helminth infections: ascariasis, trichuriasis, and hookworm. Lancet. 2006:367:1521-32.

31. Knopp S, Steinmann P, Keiser J, Utzinger J. Nematode infections: soiltransmitted helminths and Trichinella. Infect Dis Clin North Am. 2012;26:341-58.

\section{Submit your next manuscript to BioMed Central and take full advantage of:}

- Convenient online submission

- Thorough peer review

- No space constraints or color figure charges

- Immediate publication on acceptance

- Inclusion in PubMed, CAS, Scopus and Google Scholar

- Research which is freely available for redistribution 